\title{
Uso de herramientas tecnológicas para la enseñanza de la ciencias en educación media diversificada de acuerdo a la modalidad de estudio a distancia
}

\section{Use of technological tools for science teaching in diversified secondary education according to the distance learning modality}

\footnotetext{
Mervin Javier Rizales-Semprum ${ }^{\mathrm{a}^{*}}$, Carlos Luis Gómez-Valderrama ${ }^{\mathrm{b}}$, Cesar Augusto Hernández-Suarez

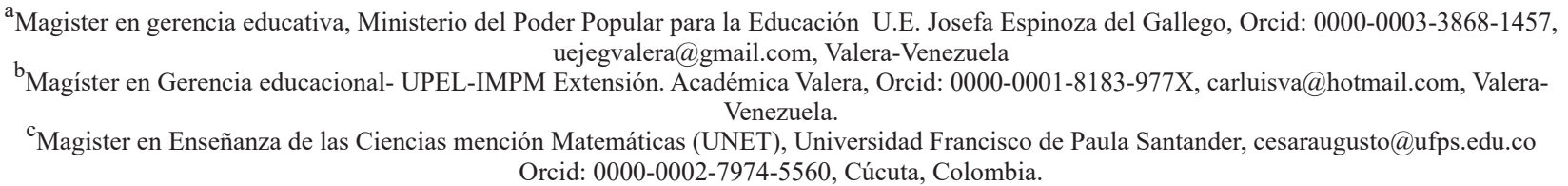

Forma de citar: Rizales-Semprum, M.J, Gómez-Valderrama, C.L \& Hernández-Suarez, C. A. Uso de herramientas tecnológicas para la enseñanza de la ciencias en educación media diversificada de acuerdo a la modalidad de estudio a distancia. Eco Matemático, 10 (2), 35-46

Recibido: 16 febrero de 2019

Aceptado: 25 abril 2019

\section{Palabras clave}

Herramienta tecnológica, enseñanza, educación, modalidad a distancias
Resumen: Se tiene como objetivo determinar en los docentes de ciencias, en un Liceo Bolivariano del estado Trujillo - Venezuela, el uso de una plataforma tecnológica en la educación media diversificada de acuerdo con la modalidad a distancia. El método, se desarrolló bajo un enfoque cuantitativo, de tipo descriptivo y diseño de campo. La muestra estuvo conformada por ocho docentes que imparten enseñanza de las ciencias. La información fue recolectada a través de un cuestionario. Se comprobó que el 37,5\% de los encuestados si sabe utilizar la plataforma Moodle. Otro $25 \%$ dice que algunas veces sabe utilizar la plataforma. Igualmente, el $75 \%$ de los encuestados perciben adecuada la modalidad a distancia para la enseñanza aprendizaje del tema. Se evidencio que un grupo significativo de docentes desconocen la plataforma Moodle y con ello su utilidad en el proceso de enseñanza aprendizaje específicamente en la modalidad de educación interactiva a distancia.

*Autor para correspondencia: cesaraugusto@ufps.edu.co 


\section{Keywords}

Technological tool, teaching, education, distance mode

\section{Introducción}

La revolución científica y tecnológica se encuentra presente en todos los ámbitos de desempeño del ser humano, se dan transformaciones en los paradigmas y sistemas de producción de la ciencia y la tecnología; sobre todo se evidencia su influencia en los cambios que deben generarse en los procesos de enseñanza-aprendizaje en los distintos niveles educativos.

La enseñanza tiene un carácter estratégico para el desarrollo de un país, de allí el reto planteado al sistema educativo de formar personas preparadas requiere favorecer "la autonomía de los estudiantes, pudiéndose adaptar a las necesidades y demandas de cada alumno o alumna de forma personalizada" (Cabero, 2014, p.23). Debe estar orientada hacia la motivación de los estudiantes, para crear experiencias de aprendizaje a nivel educativo, familiar, social y cultural sino también en lo científico. Para lograr esto, el docente estimula a los estudiantes a través de la innovación en las estrategias para una construcción significativa que pueda servir en la resolución de actividades (Gómez-Valderrama y Ramírez-Sánchez, 2017) y, ser incorporada a su campo experiencial, a fin de que, en el caso de las asignaturas de ciencias (biología, física y química), sea parte de su saber facilitando así el aprendizaje.
De acuerdo a Claxton (2003, p.34) "una de las críticas más frecuentemente esgrimidas desde la didáctica de las ciencias es que en la formación de los docentes de ciencias, se ha añadido sólo recientemente a la tradicional demanda de conocimientos científicos,", destacándose la separación de estrategias de enseñanza determinadas por la especificidad de los contenidos a enseñar.

Acosta y García (2012, p.70) sostienen que las estrategias de enseñanza "consiste esencialmente en proporcionar apoyo a la actividad constructiva de los alumnos", trabajan de manera conjunta para lograr impartir a los estudiantes los conocimientos necesarios para su desarrollo personal, educativo y profesional, al otorgarle herramientas de estudio que los orienten y ayuden de forma sucesiva a lo largo de la vida. Además, facilitan a los estudiantes los procedimiento o recursos utilizados por quien enseña para suscitar el aprendizaje (Orellana, 2008) y los contenidos nuevos a través de diversos medios para que este logre captarlos y comprenderlos dentro de un contexto.

Pues, es aquí en donde el estudiante intenta captar y retener todos los conocimientos impartidos por el docente a través de los medios y/o estrategias 
que este utilice. En el caso, de las ciencias, muchas veces los estudios se han se orientando a las motivaciones de los estudiantes de secundaria de biología, física y química, y la influencia de las metodologías de enseñanza en su interés (Méndez, 2015). Así como también, la evaluación de software educativo empleado en la enseñanza de física (Navarro, Juez, Infante y Hurtado, 2012). De allí, que haya un criterio de selección de la tecnología y estrategias didáctica(Rivero, Gómezy Abrego, 2013, p.190). No obstante, otros estudios se han dirigido al uso de herramientas tecnológicas interactivas a distancia (Arias, Sandía y Mora, 2012).

La modalidad educativa a distancia se presenta en todos los niveles de estudio, en los cuales se considera al estudiante como un sujeto autónomo, comprometido con su propio crecimiento intelectual, el cual usa la tecnología como medio de búsqueda de información y comunicación, que estudia desde su casa o espacio interactivo con flexibilidad de horario, donde organiza su tiempo por sí mismo, con la ayuda de materiales multimedia como contenidos didácticos, la biblioteca, el entorno de comunicación y con apoyo de estrategias y orientación de tutores, teniendo en cuenta el plan de estudio del docente responsable de la asignatura.

Sin embargo, en Venezuela, la educación a distancia ha sido una modalidad poco usada por las instituciones educativas en educación media diversificada, puesto que estas prefieren arraigarse a las maneras tradicionales de enseñanza-aprendizaje, pero, pese a ello, el Estado ha ofrecido diversos programas con los cuales se trata de incorporar el uso de tecnología en los niveles medio, básico y diversificado al otorgar herramientas que permitan su agregación. Por ejemplo, el proyecto Canaima como uso en la estrategias pedagógicas como recurso de enseñanza (Silva y Gómez, 2014), es por ello que han intentado ofrecer a los estudiantes de los niveles diversificados con ayuda de los Centros Bolivarianos de Información y Tecnología (CBIT), un acercamiento a la modalidad de estudio a distancia aunque no de manera sistematizada por parte de los docentes sino como uso de herramienta tecnológica y potenciar los aprendizajes en los estudiantes del Subsistema de Educación Primaria, Básica y Media, mediante el uso de las TIC (Suárez y Sánchez, 2016, p.170).

Entonces, se hace evidente la falta de implementación de este tipo de experiencia de la asignatura de las ciencias con el uso de herramientas tecnológicas y modalidad a distancia en los liceos o escuelas. Lo que trae como consecuencia, que el docente y estudiantes carezcan de habilidades en el empleo de la tecnología en esta modalidad. Por lo cual, en la mayoría de los casos, produce que estos sigan trabajando con los recursos tradicionales, tales como pizarra, borrador, tiza y libros de texto, entre otros.

Aunado a ello, el cambio en la modalidad en cómo estos docentes imparten las clases (presencial a virtual), puede generar desconfianza o miedo al ser un terreno desconocido por cuanto su implementación se convierte en un camino empinado día tras día. De este modo, tanto los estudiantes como los docentes tardarían en convertirse en aprendices de este mundo virtual, en una sociedad cada día más mediatizada, aunque con las bondades de ser parte de la sociedad de información y del conocimiento, para la mejora de los procesos de enseñanza-aprendizaje y gestión de los entornos educativos en general, facilitan la colaboración entre las familias, los centros educativos, el mundo laboral, la comunicación, entre otros, proporcionan medios para hacer llegar la formación a medida que la sociedad se la exija a cada ciudadano y también contribuir a superar desigualdades sociales.

Por otro lado, se pretende reforzar los conocimientos acerca de la enseñanza-aprendizaje de las ciencias utilizando la modalidad a distancia, específicamente, en los liceos bolivarianos. Para ello se requiere de un docente que asuma el proceso de enseñanza-aprendizaje incorporando la tecnología a 
su praxis pedagógica (Gamboa-Suárez, HernándezSuárez y Prada-Núñez, 2018).

Por consiguiente, este artículo propone un avance en torno al campo científico referente al uso de herramientas tecnológicas en cuanto a la modalidad de estudio a distancia en la educación media diversificada. Por tanto, los docentes de media diversificada tienen la oportunidad de apropiarse al proceso de enseñanza-aprendizaje en modalidad a distancia con uso de las TIC y añaden un diferencial, que establecen el uso de herramientas tecnológicas que "constituye un espacio, una herramienta y/o recurso educativo y formativo" (Tonato, 2017, p.12).

En lo práctico, examinar en los docentes, del ámbito de media diversificada, el uso de herramientas tecnológicas en la modalidad a distancia, desde este punto, significa articulaciones a las necesidades existentes del medio y propicia a la enseñanza con TIC y provocan ideas en la educación con tecnología.

De este modo, el objetivo fue determinar en los docentes de ciencias el uso de una plataforma tecnológica en la educación media diversificada de acuerdo a la modalidad a distancia.

\section{Herramientas tecnológicas en la enseñanza de las ciencias}

Saber cómo enseñar ciencias en la era digital, plantea hallar nuevas vías para un proceso pedagógico más dinámico y participativo, “prepara a las alumnas y alumnos para comprender y vivir en la globalización a que tiende el mundo de hoy" (Arteaga, Armada y Del Sol, 2016, p.172). Según las nuevas tendencias educativas, el pensamiento del sujeto que aprende adquiere un valor destacado en la relación entre docente y estudiantes. Para ello, es preciso que estos, hagan explícitas sus ideas previas sobre lo que se trata de enseñar y, por tanto, tomen conciencia de ellas. La enseñanza-aprendizaje de las ciencias tiene consecuencias muy importantes sobre la forma de organizar los contenidos en los materiales didácticos, al introducir más factores que la mera estructura lógica de las materias científicas.

Bajo en un enfoque constructivismo, las personas siempre se sitúan ante un determinado aprendizaje dotadas de ideas y concepciones previas. La mente de los estudiantes, como la de cualquier otra persona, posee una determinada estructuración conceptual que supone la existencia de auténticas teorías personales ligadas a su experiencia vital y a sus facultades cognitivas, dependientes de la edad y del estado psicoevolutivo en el que se encuentran. Así, Ausubel (1968) planteó que el núcleo central del proceso de enseñanza-aprendizaje en la insistencia sobre la importancia de conocer previamente qué sabe el estudiante antes de pretender enseñarle algo.

Como afirma Santafé-Rodríguez (2017, p. 35), la enseñanza en ciencias "requiere de grandes cambios en sus modelos de enseñanza-aprendizaje, para que los jóvenes, alcancen los estándares que se esperan...a través de procesos de formación educativa, habilidades relacionadas con el manejo del conocimiento científico en su vida cotidiana". En tal sentido, García (2001, p.75) establece que, en marco docente actual, "imparte su clase de manera tradicional, entendiéndose por ello que en su mayoría las clases son expositivas, el uso de materiales escritos como los libros de texto es utilizado como única fuente de información, los experimentos comúnmente son demostrativos, sin dar oportunidad al alumno de cuestionar y sugerir".

Por lo tanto, la enseñanza de las ciencias pasa por dejar de lado la tradicional exposición de contenidos por parte del docente para partir de la experiencia previa del estudiante y de estrategias que pueden resultar más motivante para desarrollar la enseñanza desde una perspectiva significativa, construccionista, sustentada en las habilidades cognitivas del estudiante y en su capacidad para procesar información (Salamanca-Meneses y Hernández-Suárez, 2018) y permitan promover 
el desarrollo de competencias científicas asertivas (Veloza-Rincón y Hernández-Suárez, 2018).

En correspondencia con esto, el uso de las TIC, para la enseñanza, involucra "al conjunto de procesos y productos derivados de las nuevas herramientas (hardware y software), soportes de la información relacionados con el almacenamiento, procesamiento, transmisión digitalizada de la información" (González, 2001, p.21). Además, deben contribuir con el establecimiento de una cultura digital entre los estudiantes, sin perder nunca de vista que son ellos (y no las tecnologías) el centro del proceso educativo (Hernández-Suárez, Ayala-García y Gamboa-Suárez, 2016).

Por consiguiente, todas las herramientas que permitan el almacenaje, procesamiento $\mathrm{y}$ transmisión de información de manera digitalizada pueden ser utilizadas en el contexto educativo como herramientas facilitadoras del aprendizaje de manera interactiva a distancia para la enseñanza con una serie de características tales como: "Inmaterialidad, interconexión, instantaneidad, aparición de nuevos códigos y lenguajes, elevados parámetros de calidad de imagen y sonido, digitalización, innovación, tendencia hacia la automatización, diversidad, etc." (Meneses, 2007, p.72).

De este modo, la integración de las TIC en la metodología de enseñanza abarca más que las estrategias concretas del docente en el aula. Sería un reduccionismo abordar la integración de las TIC en la enseñanza fijándose únicamente en la actuación del docente en el espacio donde se utiliza alguna tecnología. Una de las vías de superarlo es situar el estudio de esta relación TIC-metodología de enseñanza en el marco de los modelos didácticos. Éstos constituyen esquemas representativos de la realidad, que, desde fuera de la práctica, sirven para comprenderla y explicarla.

Por otro lado, las TIC deben estar inmersas en ambientes de aprendizaje colaborativos, como herramientas que apoyan el proceso activo de construcción de aprendizaje y el desarrollo de habilidades (Mercedes-Martín, Hernández-Suárez y Mendoza-Lizcano, 2017). En este contexto se concibe al aprendizaje como un proceso de construcción del conocimiento y de significado individualmente.

\section{Las TIC y plataforma tecnológica en la modalidad de estudio a distancia}

Área (2002, p. 2), considera que "cada tiempo histórico, cada civilización tiene una (o varias) tecnologías idiosincrásicas, hegemónicas para su funcionamiento". Con ello no se pretende afirmar que las tecnologías determinan las sociedades, sino que el desarrollo en un espacio preciso del tiempo se apoya en unos tipos de tecnologías. En ese sentido, las TIC, son un factor relevante que estructura, redefine, configura, las relaciones sociales, económicas, culturales, en consecuencia, la dirección del cambio social; por lo tanto, las TIC tienen tanto impacto sociocultural que condicionan el futuro desarrollo de esa sociedad como en la educación interactiva a distancia para la enseñanza de los estudiantes.

Para Montes y Ochoa (2006, p.88), las TIC fueron pensadas como medio de transmisión de información, aunque le otorga al estudiante un papel pasivo, luego fue considerada como herramienta de construcción de conocimiento, porque se identifican elementos computacionales los cuales permiten la gestión del conocimiento y otorga al estudiante un papel activo en su proceso de aprendizaje.

Las TIC dentro de un salón de clase, posibilitan nuevos modos de conocimiento, de aprendizaje y de comunicación. Así como, la interconexión y comunicar a los seres humanos en tiempo real sin importar el lugar en el cual se encuentren, a través de plataforma tecnológica en diferentes modalidades de estudios. Aunque, la TIC en el siglo XXI, son la base fundamental de los estudios a distancia. 
En esta dirección, se aclara que la plataforma tecnológica de acuerdo con Long (2004, p.86).) "define el estándar sobre el cual se desarrollan los paquetes de software". De este modo, conforman todos los elementos que permiten montar los estudios a distancia, tal es el caso, de la plataforma Moodle, que es un sistema de gestión de cursos (Course Manajemente System o CMS) para el desarrollo de una comunidad de aprendizaje, la cual cuenta con un conjunto de características para los cursos en líneas (cuestionario, talleres, foros, blogs, wikis, base de datos de actividades, encuestas, chat, glosarios y evaluaciones por pares, entre otras.).

Por otra parte, se ha dado un salto cualitativo en el desarrollo de TIC, tanto en los aspectos meramente tecnológicos, como en las posibilidades para acceder a nuevos entornos comunicativos, de información, de acceso al conocimiento en los estudios de modalidad a distancia. Se trata, así mismo, de superar y reemplazar una lógica tecnológica dirigida a aprender a usar la tecnología por sí misma, como mero instrumento ampliamente difundido, para facilitar la paulatina construcción de un discurso pedagógico en el que las TIC tengan la presencia adecuada, de acuerdo a sus posibilidades.

\section{Materiales y métodos}

La investigación se desarrolló bajo un enfoque cuantitativo, de tipo descriptivo y diseño de campo, ya que se abordó una realidad concreta en una institución educativa. Arias (2012) señala que la investigación de campo "consiste en la recolección de datos directamente de los sujetos investigados, o de la realidad donde ocurren los hechos (datos primarios), sin manipular o controlar variable alguna", y en el marco de este, se realizó un estudio descriptivo de corte transversal que se desarrolla en tres fases: Diseño de la investigación, recolección de información, análisis y desarrollo.

Fase1 - Diseño de la investigación
En esta indagación se determinó una población finita "...con características comunes para los cuales son extensivas las conclusiones de la investigación. Ésta queda delimitada por el problema y por los objetivos del estudio" Arias (2012, p. 82). La población a la cual se orientó la investigación estuvo conformada por ocho docentes de ciencias del Liceo Bolivariano Antonio José Saldivia del Municipio Carache del estado Trujillo. Mientras, que la muestra se decidió acoplar la investigación a una muestra no probabilística (Hernández, Fernández y Baptista, 2014, p.176). Por lo tanto, se selecciona como muestra a los ocho docentes la cual está integrada por 5 personas de sexo masculino $(62,5 \%)$ y 3 del sexo femenino $(37,5 \%)$. También, es necesario señalar que se observó el uso de teléfonos inteligentes. Para la organización de la investigación se realizó la recolección de información directamente en el contexto de estudio. Seguidamente y sobre la base del análisis de los resultados obtenidos sobre la modalidad interactiva a distancia, utilizando la plataforma Moodle.

\section{Fase 2 - Recolección de la información}

En la investigación se utilizó la encuesta con preguntas cerradas. Para la recolección de información, la encuesta constó de 17 preguntas que intentaron descifrar las percepciones de los docentes de ciencias sobre el uso de plataforma tecnológica Moodle en la educación media diversificada de acuerdo a la modalidad de estudio a distancia. La validez fue evaluada por expertos; se utilizó un panel de tres (03) jueces expertos en construcción, que determinaron: Pertinencia, secuencia y extensión de los ítems, con respecto a los objetivos planteados en la investigación. En cuanto a la confiabilidad fue de 0,93 para la encuesta, por ello, se llevó a cabo una prueba piloto tomando como muestra sujetos comunes a la muestra, con el fin de identificar posibles falencias en la elaboración de la encuesta. Posteriormente, se procedió a aplicar la encuesta a 
los docentes. De igual manera, en esta investigación se utilizó y apoyó en la observación para recolectar datos en cuanto la enseñanza de los docentes de ciencias.

Fase 3 - Análisis y desarrollo

Para el análisis de datos se aplicó herramientas estadísticas, para procesar "los datos obtenidos para describir, organizar, analizar e interpretar en forma apropiada los resultados" (Tamayo, 2007, p.143). De allí, una vez recolectada la información, la misma fue representada en tablas, el análisis de la misma se realizó a través de la estadística descriptiva y hoja de cálculo de Excel. Los cuales permitieron las percepciones de los docentes en cuanto al uso de herramientas tecnológicas de acuerdo a la modalidad a distancia en la media diversificada.

\section{Resultados y análisis}

En la encuesta aplicada se obtuvieron los siguientes hallazgos sobre el uso de una herramienta tecnológica en modalidad a distancia y estrategias de enseñanza-aprendizaje en educación media diversificada, la cual, se da a conocer mediante los aspectos estrategias de enseñanza -aprendizaje, conocimiento de la computadora, conocimiento de plataforma Moodle y de la modalidad a distancia. A través de los primero de ellos, se intentó identificar las estrategias de enseñanza y aprendizaje, que se detalla a continuación.

Tabla 1. Estrategias de enseñanza-aprendizaje utilizadas en las clases de ciencias.

\begin{tabular}{|c|c|c|c|c|c|c|c|}
\hline \multirow{2}{*}{\multicolumn{2}{|c|}{ Ítems }} & \multicolumn{2}{|c|}{$\mathrm{Si}$} & \multicolumn{2}{|c|}{ No } & \multicolumn{2}{|c|}{ Algunas veces } \\
\hline & & $\mathbf{F}$ & $\%$ & $\mathbf{F}$ & $\%$ & F & $\%$ \\
\hline \multirow{4}{*}{ 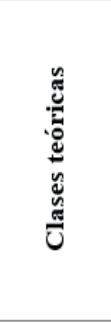 } & $\begin{array}{l}\text { 1. Utiliza como única estrategia la exposición } \\
\text { teórica }\end{array}$ & 0 & $0,0 \%$ & 0 & $0,0 \%$ & 8 & $100,0 \%$ \\
\hline & $\begin{array}{l}\text { 2. Asigna mayor importancia a los contenidos } \\
\text { de tipo conceptual }\end{array}$ & 0 & $0,0 \%$ & 0 & $0,0 \%$ & 8 & $100,0 \%$ \\
\hline & $\begin{array}{l}\text { 3. Logra el cumplimiento de los objetivos } \\
\text { propuestos a través de las estrategias utilizadas }\end{array}$ & 8 & $100,0 \%$ & 0 & $0,0 \%$ & 0 & $0,0 \%$ \\
\hline & $\begin{array}{l}\text { 4. Considera el manejo conceptual como } \\
\text { prioritario para la enseñanza aprendizaje en el } \\
\text { de ciencias. }\end{array}$ & 8 & $100,0 \%$ & 0 & $0,0 \%$ & 0 & $0,0 \%$ \\
\hline \multirow{2}{*}{ 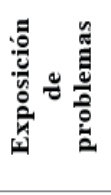 } & $\begin{array}{l}\text { 5. Refuerza la enseñanza aprendizaje utilizando } \\
\text { la resolución de problemas }\end{array}$ & 0 & $0,0 \%$ & 0 & $0,0 \%$ & 8 & $100,0 \%$ \\
\hline & $\begin{array}{l}\text { 6. Los problemas para resolver en el aula y fuera } \\
\text { de ella, y en la evaluación, se relacionan con } \\
\text { situaciones reales. }\end{array}$ & 0 & $0,0 \%$ & 0 & $0,0 \%$ & 8 & $100,0 \%$ \\
\hline 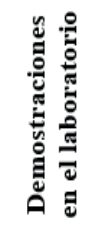 & $\begin{array}{l}\text { 7. Utiliza la demostración de fenómenos fisicos } \\
\text { en el laboratorio para facilitar la comprensión } \\
\text { del tema }\end{array}$ & 5 & $62,5 \%$ & 0 & $0,0 \%$ & 3 & $37,5 \%$ \\
\hline \multirow{3}{*}{ 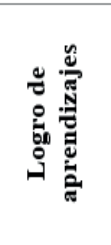 } & $\begin{array}{l}\text { 8. Los estudiantes logran comprender los } \\
\text { fenómenos fisicos con solamente las clases } \\
\text { teóricas }\end{array}$ & 0 & $0,0 \%$ & 0 & $0,0 \%$ & 8 & $100,0 \%$ \\
\hline & $\begin{array}{l}\text { 9. Sus estudiantes resuelven satisfactoriamente } \\
\text { los problemas planteados }\end{array}$ & 8 & $100,0 \%$ & 0 & $0,0 \%$ & 0 & $0,0 \%$ \\
\hline & $\begin{array}{l}\text { 10. Sus estudiantes logran identificar } \\
\text { fenómenos físicos que les ha demostrado }\end{array}$ & 0 & $0,0 \%$ & 8 & $100,0 \%$ & 0 & $0,0 \%$ \\
\hline
\end{tabular}


En la tabla 1 se reflejan los resultados obtenidos en el cuestionario aplicado a los docentes para conocer las estrategias de enseñanza-aprendizaje utilizadas en las clases de ciencias. De allí, los ítems 1, 2, 3 y 4, dan cuenta al indicador: Clases teóricas. De este modo, se evidenció que en el ítem 1, el 100\% de los docentes algunas veces utilizan como única técnica la exposición teórica; en cuanto al ítem 2, el $100 \%$ de los encuestados señalaron que algunas veces asignan mayor importancia a los contenidos de tipo conceptual. Con respecto al ítem 3, el 100\% de los docentes consideró que si logran el cumplimiento de los objetivos propuestos a través de las estrategias utilizadas. Asimismo, se comprobó en el ítem 4, que $100 \%$ de los encuestados consideran el manejo conceptual como prioritario para la enseñanza - aprendizaje de la materia en el de ciencias. Adicionalmente, en la observación realizada por los investigadores se pudo establecer que el 100\% de los docentes estudiados desarrollan sus clases a través de exposiciones magistrales de contenidos teóricos.

Al contrastar estos resultados con el basamento teórico de la investigación, se puede establecer que los docentes estudiados siguen haciendo uso de las clases teóricas como estrategia de enseñanza, lo que se corresponde con lo expresado por García (2001) imparte su clase de manera tradicional con clases son expositivas. En oposición con lo anterior, el éxito de las clases depende en gran parte de la participación que se logre de los estudiantes, la cual será posible si se cuenta con un ambiente y situaciones educativas propicias que permitan que los contenidos sean transmitidos con eficacia, sin centrarse únicamente en el discurso del docente.

También, en la tabla 2, se da cuenta sobre la exposición de problemas en el aula, donde se comprobó en el ítem 5, que el 100\% de los docentes encuestados señalaron que algunas veces refuerzan la enseñanza aprendizaje utilizando la resolución de problemas. En cuanto al ítem 6, se encontró que el $100 \%$ de los encuestados algunas veces los planteamientos de los problemas a resolver se relacionan con situaciones reales. Esta situación que también fue registrada por los investigadores en la observación realizada.

En lo relacionado al ítem 7, se demostró que el $62,5 \%$ de los docentes si utilizan la demostración de fenómenos físicos en el laboratorio para facilitar la comprensión del tema. Mientras que un $37,5 \%$, señala que algunas veces si lo hace. Esto se evidenció también en la observación realizada en la cual se pudo establecer que algunas veces los docentes realizan demostraciones de los fenómenos físicos, aunque, cabe destacar que en esta institución no existe laboratorio, por lo tanto, las demostraciones cuando se realizan se hacen en el aula de clases.

Lo anterior, soporta con lo establecido por Santafé-Rodríguez (2017) en cuanto a que en la enseñanza en ciencias requiere de grandes cambios en sus modelos de enseñanza-aprendizaje para desarrollar y aplicar ideas importantes (principios y leyes) que expliquen un amplio campo de fenómenos a través de procesos de formación educativa, habilidades relacionadas con el manejo del conocimiento científico en su vida cotidiana.

De igual modo, se determinó que sobre las percepciones de los docentes en cuánto al logro de aprendizaje, referenciados a los ítems 8, 9 y 10. De este modo, se pudo verificar en el ítem 8 , que el $100 \%$ de los docentes de ciencias algunas veces perciben que los estudiantes logran comprender los fenómenos físicos con solamente las clases teóricas. En cuanto al ítem 9, se determinó que $100 \%$, piensan que algunas veces los estudiantes resuelven satisfactoriamente los problemas planteados. De la misma forma, se confirmó en el ítem 10, que el 100\% de los docentes de ciencias no creen que sus estudiantes logren identificar fenómenos físicos que les ha demostrado.

Estos resultados (ítem, 8, 9 y 10) se contraponen el planteamiento de Acosta y García (2012, p.70) 
quienes mantienen la idea de que las estrategias de enseñanza deben proporcionar andamiaje a la actividad constructiva de los estudiantes, en los medios o recursos utilizados por quien enseña para suscitar el aprendizaje (Orellana, 2008) e incitar a los estudiantes a través de la innovación en las estrategias para una construcción significativa que pueda servir en la resolución de actividades (GómezValderrama y Ramírez-Sánchez, 2017).

Tabla 2. Conocimiento sobre la utilización de la computadora

\begin{tabular}{lccccccc}
\hline \multirow{2}{*}{ Items } & \multicolumn{2}{c}{$\mathrm{Si}$} & \multicolumn{2}{c}{$\mathrm{N}_{0}$} & \multicolumn{2}{c}{ Algunas veces } \\
\cline { 2 - 8 } & $\mathrm{F}$ & $\%$ & $\mathrm{~F}$ & $\%$ & $\mathrm{~F}$ & $\%$ \\
\hline 11. Conoce el funcionamiento de una computadora & 8 & $100 \%$ & $\cdot$ & $\cdot$ & $\cdot$ & - \\
\hline $\begin{array}{l}\text { 12. Conoce programas en computadora para desarrollar el tema } \\
\text { durante las clases }\end{array}$ & 4 & $50 \%$ & 3 & $37,5 \%$ & 1 & $12,5 \%$ \\
\hline
\end{tabular}

La tabla 2, hace referencia a los ítems 11 y 12 , que da cuenta sobre el conocimiento que tienen los docentes de ciencias del del Liceo Bolivariano Antonio José Saldivia del Municipio Carache del estado Trujillo. Donde se logró comprobar que en el ítem 11, el $100 \%$ de los encuestados conocen el funcionamiento de una computadora. Mientras que para el ítem 12, se verificó que el $50 \%$ de los encuestados si conocen programas en computadora para desarrollar el tema durante las clases, de otro lado, un $37,5 \%$ dice que no conoce y un $12,5 \%$ señalaron que algunas veces conocen programas para desarrollar el tema durante las clases.

Atendiendo los ítems 11 y 12, se evidencia que los docentes de ciencias pudieran parcialmente tener un criterio de selección de la tecnología y estrategias didácticas (Rivero, Gómez y Abrego, 2013), para preparar los estudiantes a comprender y vivir en la globalización del conocimiento que tiende el mundo de hoy (Arteaga, Armada y Del Sol, 2016).

Tabla 3. Conocimiento de la plataforma Moodle

\begin{tabular}{|c|c|c|c|c|c|c|}
\hline \multirow{2}{*}{ Items } & \multicolumn{2}{|c|}{$\mathrm{Si}$} & \multicolumn{2}{|c|}{$\mathrm{N}_{0}$} & \multicolumn{2}{|c|}{ Algunas reces } \\
\hline & F & $\%$ & $\mathrm{~F}$ & $\%$ & $\mathrm{~F}$ & $\%$ \\
\hline 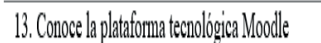 & 6 & $75 \%$ & 2 & $25 \%$ & . & . \\
\hline 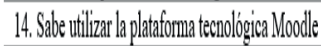 & 3 & $37,5 \%$ & 3. & $37,9 \%$ & 2 & $25 \%$ \\
\hline
\end{tabular}

En la tabla 3, se muestran los datos obtenidos en los ítems 13 y 14, que dan cuenta sobre el conocimiento que tienen los docentes sobre la plataforma Moodle. Donde se identificó que el 75\% de los encuestados conocen la plataforma Moodle, el otro $25 \%$ dice que no. Con respecto al ítem 14 , se comprobó que el $37,5 \%$ de los encuestados si sabe utilizar la plataforma Moodle. Otro $25 \%$ dice que algunas veces sabe utilizar la plataforma y el resto un $37,5 \%$ dice que no sabe utilizar dicha plataforma. Por lo tanto, los docentes incluidos en la investigación desconocen parcialmente a Moodle, como sistema de gestión de cursos que soporta a un marco de educación social constructivista. Aunque la falta de conocimiento pudiera dar papel pasivo a los estudiantes al querer utilizar la plataforma como tecnología de información y comunicación como medio de conocimiento y transmisión de información (Montes y Ochoa, 2006, p.88) porque se identifican elementos computacionales que poco favorecen el uso de la plataforma para que los docentes, pudiéndose adaptarse a las nuevas realidades, necesidades y demandas de cada estudiante de forma personalizada (Cabero, 2014).

Tabla 4. Conocimiento de los docentes sobre la modalidad a distancia

\begin{tabular}{lcccccc}
\hline \multirow{2}{*}{ İtems } & \multicolumn{2}{c}{$\mathrm{Si}$} & \multicolumn{2}{c}{ No } & \multicolumn{2}{c}{ Algunas veces } \\
\cline { 2 - 7 } & $\mathrm{F}$ & $\%$ & $\mathrm{~F}$ & $\%$ & $\mathrm{~F}$ & $\%$ \\
\hline $\begin{array}{l}\text { 15. Ha logrado utilizar el computador para desarrollar clases } \\
\text { distancia }\end{array}$ & 2 & $25 \%$ & 5 & $62,5 \%$ & 1 & $12,5 \%$ \\
\hline $\begin{array}{l}\text { 16. Considera la plataforma Moodle adecuada para la enseñanza del } \\
\text { tema a distancia }\end{array}$ & 6 & $75 \%$ & 1 & 12,5 & 1 & $12,5 \%$ \\
\hline $\begin{array}{l}\text { 17. Considera adecuada la modalidad a distancia para la enseñanza } \\
\text { aprendizaje del tema }\end{array}$ & 6 & $75 \%$ & 2 & $25 \%$ & 0 & $0 \%$ \\
\hline \hline
\end{tabular}

La tabla 4 indica sobre el conocimiento de los docentes sobre la modalidad a distancia. En el cual, se conoció en el ítem 15 , que el $62,5 \%$ de los docentes encuestados coincidieron en señalar que no han logrado utilizar el computador para desarrollar clases a distancia, aunque, un $25 \%$ dice que si y un $12,5 \%$ dice que algunas veces. En relación al ítem 16 , se determinó que el $75 \%$ si considera adecuada la plataforma Moodle adecuada para la enseñanza del tema a distancia. El resto, dicen que no $(12,5 \%)$ $\mathrm{y}$ otros que algunas veces (12,5\%) Moodle es adecuado para la enseñanza a distancia. En cuanto 
al ítem 17 , se verificó que $75 \%$ de los encuestados perciben adecuada la modalidad a distancia para la enseñanza aprendizaje del tema. El otro $25 \%$ considera que no es adecuada. Es necesario destacar, que de acuerdo a la observación realizada por los investigadores algunas veces incorporan las computadoras u otra herramienta de las tecnologías y comunicación en el desarrollo de sus clases. En tal sentido, siguen asumiendo una metodología tradicional de enseñanza más centrada en transmitir información, descartando el uso de la tecnología como recurso instruccional de apoyo al proceso de enseñanza aprendizaje.

En correspondencia con estos resultados, se debe destacar, que no todos los docentes estudiados comprenden la importancia de utilizar la plataforma Moodle y la educación a distancia para la enseñanza, la cual permite crear cuestionarios, chats, foros, videos, blogs, consultar material, todo lo cual facilita el aprendizaje de este contenido. Es decir, los docentes están subvalorando el alcance pedagógico de los recursos que ofrece la plataforma y no han visualizado los beneficios que traen dentro del proceso de formación, de acuerdo con lo expresado por Prada-Núñez, Hernández-Suárez y Gamboa-Suárez (2019). Por lo que puede señalarse, que los docentes que no entienden la utilidad de esta modalidad es porque desconocen que ésta les permite aprender en los entornos virtuales, modalidades educativas que apuntan a ampliar el acceso a la educación y a la formación, liberando a los docentes y estudiantes de las limitaciones de tiempo y espacio, y ofreciendo mayor flexibilidad en las oportunidades de aprendizaje individual o grupal, así como un espacio que apoya el desarrollo de habilidades (Mercedes-Martín, HernándezSuárez y Mendoza-Lizcano, 2017).

\section{Conclusiones}

Los resultados obtenidos en la investigación, permitieron establecer las siguientes conclusiones:
Los docentes de la asignatura de ciencias suelen utilizar las clases teóricas para el aprendizaje, si bien utilizan la resolución de problemas, las demostraciones de los fenómenos físicos son ocasionalmente presentadas por cuanto la institución carece de laboratorio con los materiales necesarios para realizar tales demostraciones. Por lo tanto, suelen centrar su actividad de enseñanza y el proceso de aprendizaje en las clases magistrales donde explican los conceptos y en la resolución de ejercicios que les permitan a los estudiantes poner en práctica los conceptos presentados. Por otra parte, las estrategias utilizadas permiten que los estudiantes parcialmente logren aprender los contenidos desarrollados a través de la resolución de problemas y del reconocimiento de los fenómenos físicos.

Aunado a esto, los docentes encuestados a pesar de que tienen un dominio básico del computador, en algunos casos desconocen los programas que pueden ayudarlos a desarrollar su actividad pedagógica, así como también, desconocen la plataforma Moodle y con ello su utilidad en el proceso de enseñanza aprendizaje específicamente en la modalidad de educación interactiva a distancia. Esta última, es también desconocida para un grupo significativo de los docentes incluidos en el estudio y, por ende, desconocen su utilidad para la enseñanza aprendizaje; aunque ocasionalmente han incorporado el uso de algún tipo de tecnología en sus actividades de aula.

\section{Referencias}

Acosta, F., \& García, M. (2012). Estrategias de enseñanza utilizadas por los docentes de biología en las universidades públicas. Omnia, 18(2),67-82. https://www.redalyc.org/ pdf/737/73723402005.pdf

Área, M. (2002). Sociedad de la Información, Tecnologías Digitales y Educación. http:// tecnologiaedu.us.es/bibliovir/publico0.htm

Arias, F. (2012) El Proyecto de Investigación Introducción a la metodología científica. 6ta. Edición. Editorial EPISTEME, C.A. Caracas - 
República Bolivariana de Venezuela

Arias, M. y Sandia, B. y Mora, E. (2012). La didáctica y las herramientas tecnológicas web en la educación interactiva a distancia. Educere, 16 (53), 21-36. https://www.redalyc.org/articulo. oa? $\mathrm{id}=356 / 35623538004$

Arteaga, E., Armada, L., y Del Sol, J. (2016). Teaching science in the new millennium. Challenges and suggestions. Revista Universidad y Sociedad, 8(1), 169-176. http://scielo.sld. $\mathrm{cu} /$ scielo.php?script $=$ sci_arttext\&pid $=\mathrm{S} 2218$ 36202016000100025\&lng=es\&tlng=en.

Ausubel. D. P. (1968). Educational Psychology: A Cognitive View. New York: Holt. Rinehart \& Winston.

Cabero, J. (2014). Reflexiones sobre la brecha digital y la educación: siguiendo el debate. Inmanencia, 4(2), 14-26. http://ppct.caicyt.gov.ar/index.php/ inmanencia/article/view/6242

Claxton, G. (2003). Educar mentes curiosas. Madrid: Visor.

Gamboa-Suárez, A., Hernández-Suárez, C. y Prada-Núñez, R. (2018). Práctica pedagógica y competencias TIC. Saber, Ciencia y Libertad, 13(1), 258-274. https://doi.org/10.18041/23823240/saber.2018v13n1.2090

García, M. (2001). Las actividades experimentales en la escuela secundaria. Perfiles educativos, 23(94), 70-90. http://www.scielo.org.mx/ scielo.php?script $=$ sci_arttext\&pid $=$ S 0185 26982001000400005\&lng=es\&tlng=es.

Gómez-Valderrama, C. y Ramírez-Sánchez, R. (2017). Comunidades de aprendizaje móvil, Mastery Learning y el problema 2 sigma como estrategias para el conocimiento previo, en la resolución de actividades. Ecomatemático, 8, 25-32. https://doi.org/10.22463/17948231.1376

Tonato, M. (2017). El docente y el uso de herramientas tecnológicas de enseñanzaaprendizaje en la Educación Básica Ecuatoriana. Journal of Science and Research, 2(7), 1014. https://doi.org/10.26910/issn.25288083vol2iss7.2017pp10-14González, A (2001). Tecnología Digital. Colombia: Mc Graw Hill.
Hernández, R., Fernández, C y Baptista, M. (2014). Metodología de la Investigación. McGraw-Hill/ Interamericana

Hernández-Suárez, C., Ayala-García, E. y GamboaSuárez, A. (2016). Modelo de competencias TIC para docentes: Una propuesta para la construcción de contextos educativos innovadores y la consolidación de aprendizajes en educación superior. Katharsis: Revista de Ciencias Sociales, (22), 221-265. https://doi. org/10.25057/25005731.821

Long, L. (2004). Introducción a las Computadoras y a los Sistemas de Información. México: Prentice Hall.

Méndez, D. (2015). Estudio de las motivaciones de los estudiantes de secundaria de física $\mathrm{y}$ química y la influencia de las metodologías de enseñanza en su interés. Educación XX1, 18 (2), 215-235. https://www.redalyc.org/articulo. oa? id=706/70638708009

Meneses, G. (2007). Las nuevas tecnologías de la información (tesis, Universitat rovira i virgili). https:/www.tesisenred.net/bitstream/e/10803/ 8929/2Lasnuevastecnologiasdelainformacion. pdf? sequence $=8$

Mercedes- Martín, M., Hernández-Suárez, C. y Mendoza-Lizcano, S. (2017). Ambientes de aprendizaje basados en herramientas web para el desarrollo de competencias TIC en la docencia. Perspectivas, 2(1). 97-104. https:// doi.org/10.22463/25909215.1282

Montes, J. y Ochoa, S. (2006). Apropiación de las tecnologías de la información y comunicación en cursos universitarios. Acta Colombiana de Psicología, 9 (2), 87-100. https://www.redalyc. org/articulo.oa? id=798/79890209

Navarro; J., Juez, J., Infante, E. y Hurtado, A (2012). Elementos fundamentales para la evaluación objetiva de software empleado en la enseñanza de la física. Góndola, Enseñanza y Aprendizaje de las Ciencias. 7(1), 66-75. https://revistas. udistrital.edu.co/index.php/GDLA/article/ view/5039/6666

Orellana, Arturo (2008). Estrategias en Educación. 
Venezuela: Ediciones Mc. Graw Hill

Prada Núñez, R., Hernández Suárez, C. A. y Gamboa, A. A. (2019). Usos y efectos de la implementación de una plataforma digital en el proceso de enseñanza de futuros docentes en matemáticas. Revista Virtual Universidad Católica del Norte, (57), 137-156. https://doi. org/10.35575/rvucn.n57a10

Rivero, I., Gómez, M. y Abrego, R (2013). Tecnologías educativas y estrategias didácticas: criterios de selección. Revista Educación y Tecnología, 3, 190-206. http://revistas.umce.cl/ index.php/edytec/article/view/134

Salamanca-Meneses, X. y Hernández-Suárez, C. A. (2018). Enseñanza en ciencias: la investigación como estrategia pedagógica. trilogía Ciencia Tecnología Sociedad, 10(19), 133-148. https:// doi.org/10.22430/21457778.1025

Santafé, Y. (2017). Fortalecimiento de competencias científicas en la asignatura de física para estudiantes de undécimo grado en Colombia. Ecomatemático, 8(1). 34-42. https://doi. org/10.22463/17948231.1473

Silva, C. y Gómez, N. (2014). Estrategias pedagógicas dirigidas a los docentes para la aplicación del proyecto Canaima educativo como recurso de enseñanza. Sustentabilidad al dia, 1. http://www.revencyt.ula.ve/storage/repo/ ArchivoDocumento/resustenta/n3/art02.pdf

Suárez, N. y Sánchez, B (2016). Implicaciones del proyecto Canaima I en las formas de apropiación de la lectura y escritura en los niños y niñas del $1^{\circ}$ grado "a" de la E.B. "Antonio Ricaurte". ARJÉ. Revista de Postgrado FaCE-UC, 10(19), 166-180. http://arje.bc.uc.edu.ve/arj19/art13.pdf

Tamayo, M. (2007). El Proceso de la Investigación Científica: Incluye evaluación y administración de proyectos de investigación. México: Limusa. Veloza-Rincón, R. y Hernández-Suárez, C. (2018). Valoración de las estrategias adoptadas por docentes en la enseñanza de la ciencia desde la perspectiva de los estudiantes de educación básica. Ánfora, 25(45), 43-69. https://doi. org/10.30854/anf.v25.n45.2018.512 